\title{
The correlation of maternal and fetal heart rates - an important aspect of CTG interpretation
}

\author{
Joachim H Nagel
}

\section{Introduction}

Although cardiotocography (CTG) is the most commonly used method in perinatal monitoring, there are still some difficulties surrounding its interpretation. In many cases an unequivocal diagnosis is not possible without additional physiological information. The reasons may be either physiological or due to shortcomings of existing instrumentation. A typical example is shown in Figure 1, where the CTG reveals no reason for the acceleration of the fetal heart rate (FHR). Uterine activity is completely absent, and no fetal movement is detectable. Considering, however, the maternal heart rate (MHR), the acceleration becomes intelligible as a result of feto-maternal coupling.

A most serious situation is documented in Figure 2. There is a quite normal CTG, no uterine activity and an FHR curve of rather good quality that reveals no abnormalities. But the simultaneously recorded fetal ECG shows severe arrhythmia. Probably no existing fetal monitor would have been able to detect this condition. We were able to recognize the arrhythmia because we monitored not only the fetal but also the maternal heart rate, which was found to be markedly abnormal. Our interpretation of the feto-maternal coupling indicated that a direct influence on the fetal cardiac system was to be expected, so we recorded the fetal ECG in order to check the inconspicuous FHR curve.

\section{Interaction between maternal and fetal heart rate}

\section{Oxygen availability}

The question arises as to whether or not the correlation between FHR and MHR represents a random event. We feel sure that there is a systematic dependence. Fetal oxygen availability is a direct function of maternal cardiac output and oxygen saturation, as well as of placental sufficiency. It is well known that a reduction in available oxygen usually influences the fetal heart rate through the action of the cardiac regulatory system, which tends to compensate for the oxygen deficit by accelerating the heart to ensure an adequate supply to fetal tissues. As a consequence, both placental insufficiency and changes in maternal blood supply may influence FHR. 


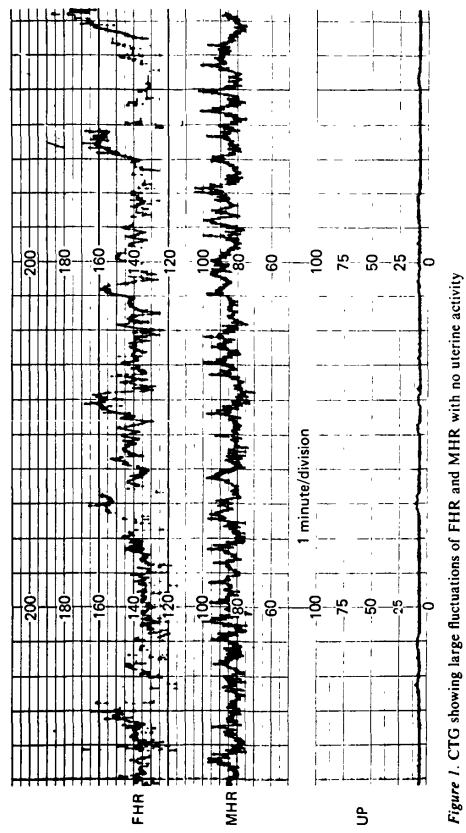



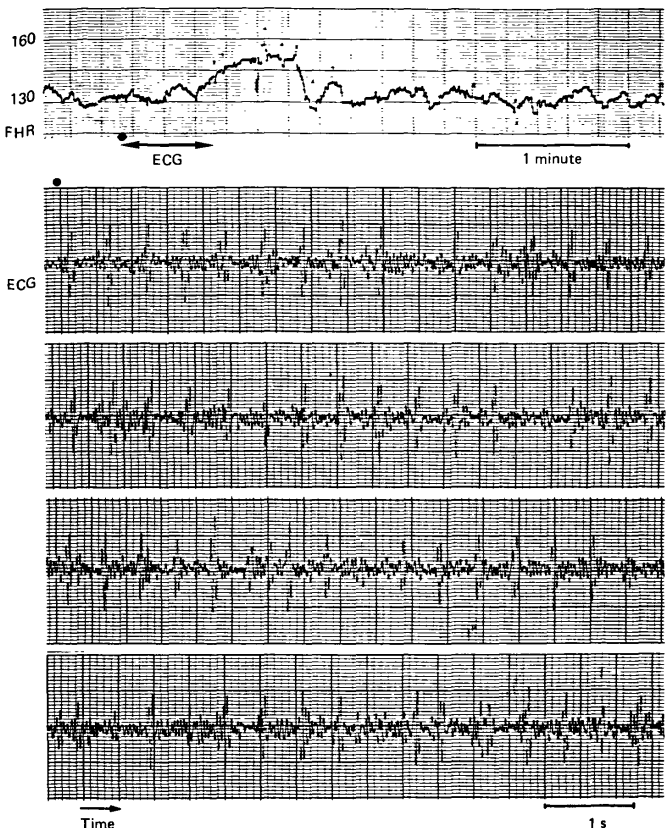

Figure 2. Fetal arrhythmia hidden by an inconspicuous FHR curve

This reasoning is not new, but what is important is to draw the right conclusions. CTG interpretation is usually based on correlations between FHR and uterine pressure. But what is to be done in the absence of pressure changes, or if an abnormal pattern presents during labour? Can we be sure that the increasing uterine pressure is the reason for heart rate variations in every case? And what influence has the maternal cardiovascular system?

Let us consider the physiological situation. Labour activity represents a stress not only for the fetus, but also for the mother, resulting in maternal heart rate variations 

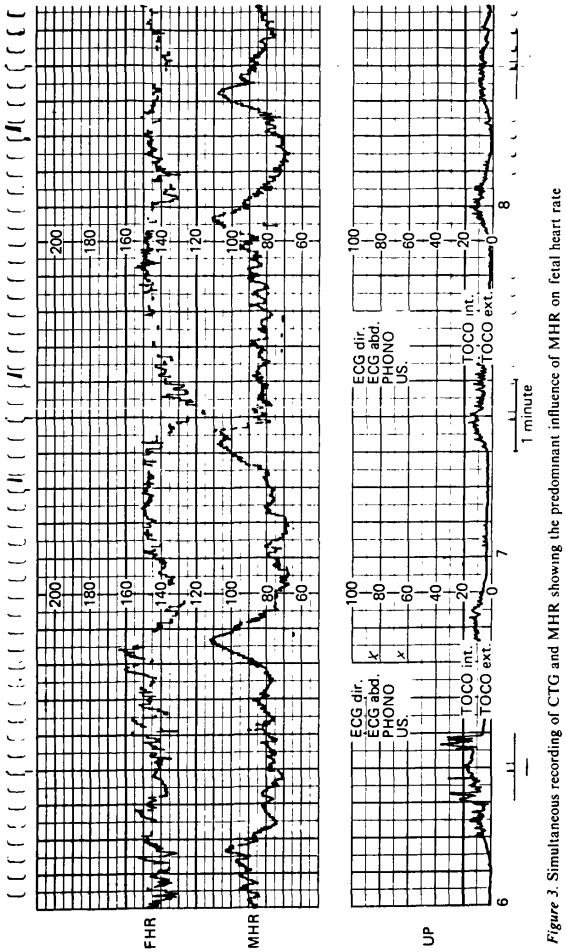

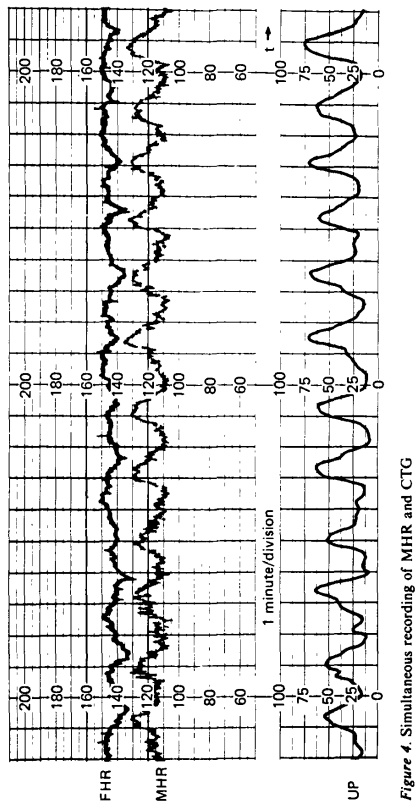
and intensified respiration. Some authors have already demonstrated an augmented $\mathrm{PO}_{2}$ during labour. With normal materral cardiac output and placental sufficiency this means an increase in the fetal oxygen supply. The physiological reaction will be a deceleration of fetal heart rate, as is, indeed, observed. On the other hand, fetal stress caused by uterine compressions would, as a normal physiological reaction, result in an acceleration comparable to that of the maternal heart. Obviously there are two opposing regulatory processes. So should we not logically attribute the commonly observed decelerations to the maternal cardiovascular system rather than to uterine pressure? An obvious case is shown in Figure 3. The normal reaction to labour activity is an accleration of MHR and a 'dip' in the FHR. The first contraction shown in Figure 3 does not produce a maternal response, and thus there is no deceleration of FHR.

\section{Interpretation of the timing of FHR 'dips'}

A second point of interest is the interpretation of the onset of the 'dips'. If the previous statement holds, then we must also relate it to the changed conditions of the maternal cardiovascular system. Again, the consequences are quite surprising. With the onset of a uterine contraction we first detect a maternal reaction, expressed as a change in the heart rate. The associated increase in $\mathrm{PO}_{2}$ results in fetal heart rate deceleration. But there must be a delay vis-à-vis the acceleration of the maternal heart because the passage of oxygen through the placenta takes a certain time, which will depend upon placental efficiency. In common with umbilical cord compression, placental insufficiency retards the increase in fetal $\mathrm{PO}_{2}$ and thus heart rate deceleration. So we will detect a late deceleration. But the essential point is that late decelerations should not automatically be interpreted as placental insufficiency or any other disorder, since we have to take into account a second time constant, namely the time delay in the maternal response to uterine contraction, which may be increased without any consequences for fetal well-being. This accords with the experience of other authors who have found, without knowing the physiological reason, that a late 'dip' should not be interpreted as a sign of risk provided the FHR oscillations remain within their normal range. Therefore, we believe that the unequivocal interpretation of 'dips' would be considerably improved by a knowledge of the maternal condition.

Figures 3 and 4 show the simultaneous recording of both maternal and fetal heart rates together with uterine pressure. There is a remarkable conformity between FHR and MHR, but with an inverse relationship. Detailed analysis of the correlation between FHR and MHR, which is very simple to perform, provides important information. The correlation coefficient is highest when the maternal heart rate is delayed vis-à-vis the FHR for about 30-40 seconds. This is the response time of the placenta and the fetal regulatory system. In other words, if the maternal oxygen supply changes, it will take about 30 seconds for the FHR to respond. This is an important fact which permits an assessment of placental sufficiency by following up this time delay. Insufficiency will increase the delay and reduce the correlation.

\section{Conclusions}

The heart rate is a rather poor indicator of the physiological condition both in the fetus and in the mother. Even such simple measurements as maternal blood pressure recording can provide additional information (Figure 5). However, for an accurate 

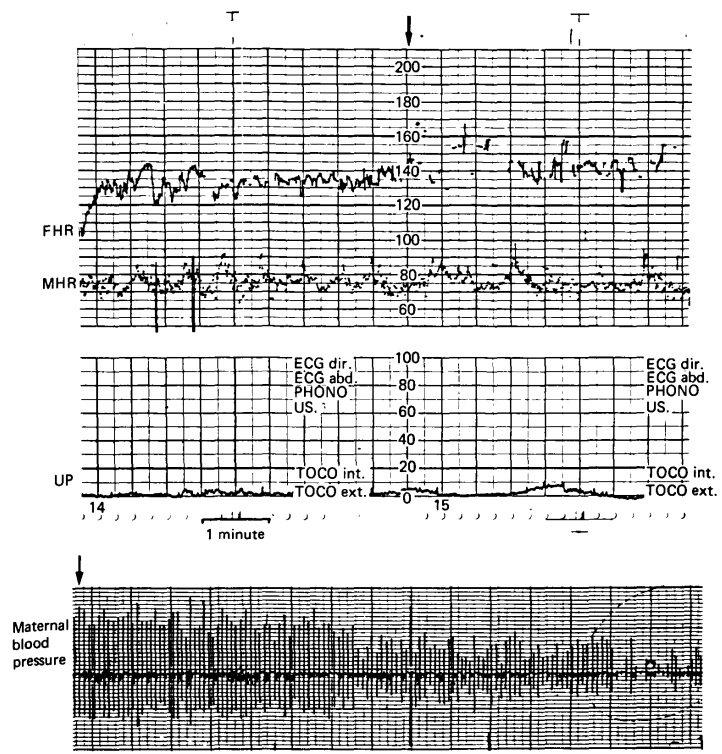

Figure 5. Fetal arrhythmia caused by a breakdown of maternal blood pressure

analysis of the feto-maternal coupling at least cardiac output and maternal oxygen saturation should be monitored. Nevertheless, our investigations have shown that for routine use even the MHR provides sufficient information to improve the interpretation of the CTG quite considerably. The correlation analysis of fetal and maternal heart rates facilitates diagnosis, particularly in borderline cases, and may help to prevent misinterpretation of the CTG. Since the provision of an additional MHR channel in perinatal monitors is very simple and inexpensive, future systems should not be without one. 